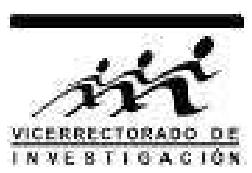

\title{
Simulación del sistema hidraúlico e hidrológico de la cuenca del río Rímac-Santa Eulalia
}

\author{
D. D. Sarango*1, J. Gástelo ${ }^{1}$, T. O. Velásquez ${ }^{1}$, C. A. Alata ${ }^{1}$ y C. A. Montalvo ${ }^{1}$ \\ ${ }^{1}$ Departamento de Ingeniería Mecánica de Fluidos, Facultad de Ciencias Físicas, Universidad Nacional Mayor \\ de San Marcos, Lima, Perú
}

Recibido 30 setiembre 2014 - Aceptado 22 octubre 2014

\begin{abstract}
El presente trabajo tiene como objetivo elaborar un modelo de simulación, para la operación del sistema hidráulico e hidrológico de la cuenca del río Rímac-Santa Eulalia, que incluye el proyecto de la central hidroeléctrica de Huanza actualmente en construcción, con los objetivos de generación de energía eléctrica, el abastecimiento de agua para uso agrícola y uso poblacional de Lima y Callao. El sistema hidráulico está formado por las centrales hidroeléctricas de EDEGEL S.A. que operan usando los caudales regulados y no regulados de la cuenca de los ríos Santa Eulalia, Rímac, cuenca de la laguna Marcapomacocha y caudales infiltrados en el Túnel Trasandino.

Se ha evaluado la operación del proyecto de la C. H. Huanza, ubicada aguas arriba del embalse Sheque, con la finalidad de no afectar la operación de C. H. Huinco propiedad de EDEGEL.

Los resultados obtenidos con el modelo de simulación son aceptables por que garantizan un caudal medio multianual de $12.51 \mathrm{~m}^{3} / \mathrm{s}$ a ser utilizado por la C.H. Huinco y permite contar en un $95 \%$ del tiempo simulado en el periodo 1978-2005 con un caudal de $25 \mathrm{~m}^{3} / \mathrm{s}$ en la estación de Chosica, para cubrir la demanda agrícola, poblacional y ecológica de las provincias de Lima y Callao.

El modelo de simulación elaborado puede ser usado por EDEGEL, ANA, MINAG, SENAMHI y SEDAPAL para poder evaluar otros proyectos hidroeléctricos, de irrigación y de abastecimiento de agua potable en la cuenca del río Rimac-Santa Eulalia.

Palabras claves: modelo de simulación, cuenca hidrográfica Rímac-Santa Eulalia, centrales hidroeléctricas, embalses, presas.
\end{abstract}

\section{Hydraulic and hydrological system simulation of the Rímac-Santa Eulalia River basin}

This research aims to develop a simulation model, for the operation of hydraulic and hydrological system of the Rima-Santa Eulalia River basin that includes the H. P. Huanza project now in construction, with purposes of power generation, water supply for agricultural use and drink water for population of Lima and Callao. The hydraulic system is formed by hydroelectric EDEGEL S.A. that operate using the regulated and unregulated flow of the Santa Eulalia river basin, Rímac river basin, Marcapomacocha Lake watershed and flows into the tunnel Trasandino infiltrators.

We evaluated the operation of the project H. P. Huanza located upstream Sheque reservoir, in order to not affect the operation of $\mathrm{H}$. P. Huinco EDEGEL property. The results obtained from the simulation model are acceptable for guaranteeing an average flow rate of $12.51 \mathrm{~m}^{3} / \mathrm{s}$ to be used by the $\mathrm{H}$. P. Huinco and we can count on $95 \%$ of simulated time period 1978-2005 with a flow rate of $25.0 \mathrm{~m}^{3} / \mathrm{s}$ in Chosica Station, to cover agricultural, population and ecological demand of Lima and Callao Provinces. The simulation model developed could be used by EDEGEL, ANA, MINAG, SEDAPAL and SENAMHI to evaluate other hydroelectric projects, irrigation and drinking water supply in the Rímac-Santa Eulalia river basin.

Keywords: Simulation model, Rímac-Santa Eulalia hydrographic watershed, hydroelectric plants, dams.

\footnotetext{
*dsarangoj@unmsm.edu.pe
} 
El sistema hidroeléctrico de EDEGEL S.A. es uno de los principales generadores de energía que cubre aproximadamente el $6 \%$ de la demanda total de energía del Sistema Eléctrico Interconectado Nacional (SEIN) de nuestro país, siendo el principal centro de demanda abastecido por este sistema el de la ciudad de Lima y Callao, el $94 \%$ restante de la demanda del SEIN es atendida por otras empresas de generación hidroeléctrica y termoeléctrica.

El sistema hidroeléctrico de EDEGEL S.A., está for- mado por las centrales Huinco (247.34 MW), Matucana (128.58 MW), Callahuanca (80.43 MW), Moyopampa (64.70 MW) y Huampani (30.18 MW) que operan usando los caudales no regulados de la cuenca de los ríos Sta. Eulalia y Rímac, lo volúmenes regulados de las 19 lagunas de la cuenca alta del río Sta. Eulalia y cuenca de la laguna Marcapomacocha, así mismo también usa los aportes no regulados de la cuenca de Marcapomacocha, y los caudales infiltrados en el túnel trasandino, ver Figura 1 y 2 [1].

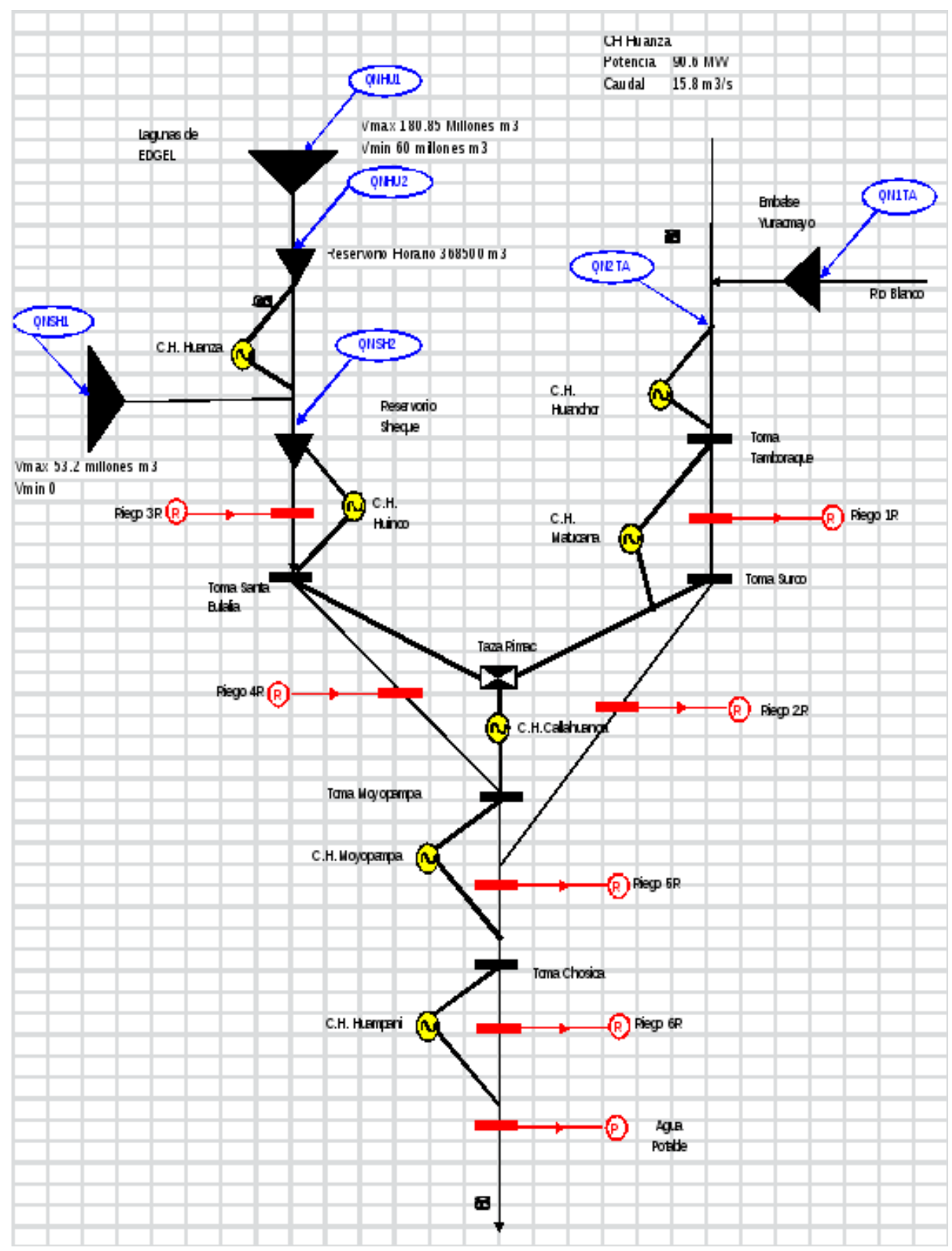

Figura 1: Diagrama topológico del sistema hidraúlico de la cuenca del río Rímac - río Santa Eulalia. Elaboración propia con datos de EDEGEL S.A. 


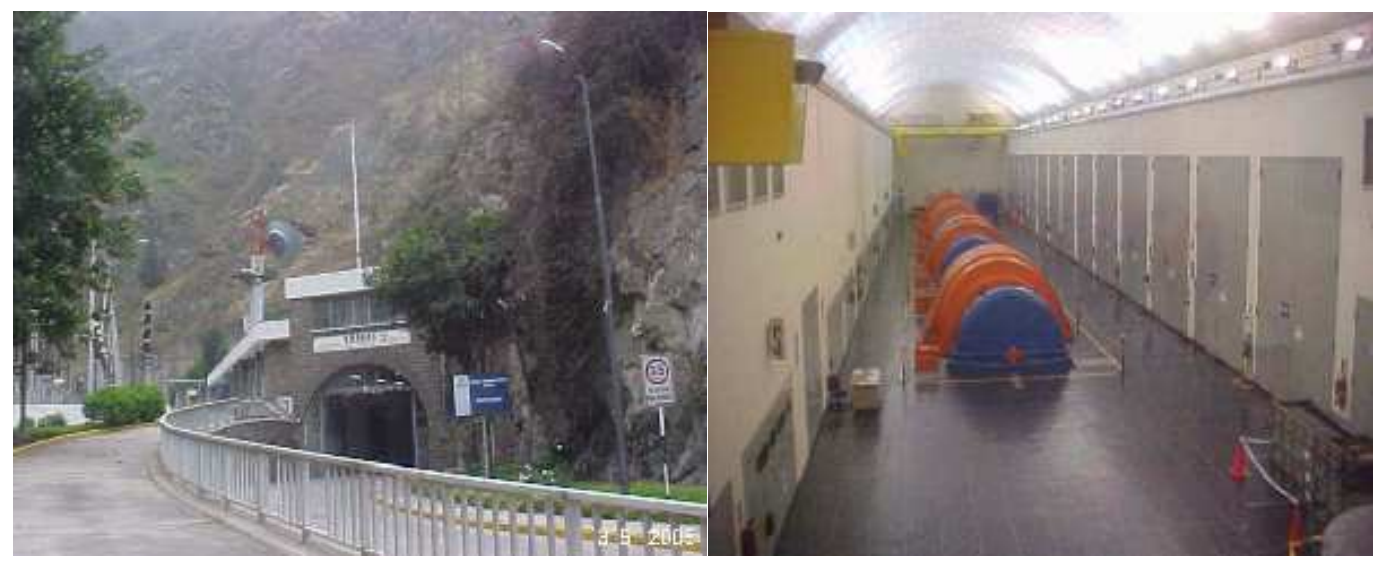

Figura 2: Central hidroeléctrica de Huinco. Fuente EDEGEL S.A.

Una restricción muy importante al sistema Rímac-Sta. Eulalia lo constituyen las demandas de agua potable de las ciudades de Lima y Callao, existen otras demandas de agua de menor escala para irrigación.

Es bajo este contexto que se desea evaluar la operación del proyecto de la C.H. Huanza (80 MW), ubicada aguas arriba del embalse Sheque, lugar donde se ubica la toma de la central hidroeléctrica Huinco.

Con este objetivo se ha evaluado las disponibilidades hídricas a ser aprovechadas por la central de Huanza, teniendo como premisa básica, que el uso de las aguas por este proyecto no alterará el régimen de la $\mathrm{C}$.H. Huinco, ni en las condiciones actuales, ni en los que establezca SEDAPAL para afianzar el sistema Marcapomacocha, con el proyecto MARCA III, que entro en operación el año 2000.

Las metas a cumplir por el sistema hidráulico e hídrico de la cuenca del río Rímac-Marcapomacocha, es el de tener $25.0 \mathrm{~m}^{3} / \mathrm{s}$ en la estación Chosica para satisfacer las necesidades de agua potable, agricultura y ecológico, y de $8.0 \mathrm{~m}^{3} / \mathrm{s}$ en Sheque-Huinco, elaborándose para esto un modelo de simulación hidráulico-hidrológico de la operación de este sistema.

\section{Definiciones}

Para el esquema de la simulación que presentamos en este trabajo debemos considerar algunas definiciones que abarcan todo el panorama del sistema en estudio.

El sistema hidráulico de la cuenca de los ríos RímacSanta Eulalia. Este sistema pertenece actualmente a la Empresa de Generación Eléctrica de Lima S.A.-EDEGEL S.A.-, y su explotación comienza desde el año 1875 mediante la regulación de las lagunas de la cuenca alta del río Sta. Eulalia, por la necesidad de satisfacer las demandas crecientes de agua, y, en el presente siglo, se efectuó obras de transvase de agua regulada desde la cuenca del río Mantaro ampliando el sistema hidrográfico y modificando su régimen de descargas.

La última regulación efectuada fue en el río Blanco, donde se ha construido el reservorio de Yuracmayo de 50 MMC de volumen total, y SEDAPAL S.A. ha implementado el proyecto de afianzamiento de MARCA III en el año 2000 para incrementar el recurso hídrico del río Rímac para el abastecimiento de agua potable para las provinicas de Lima y Callao [2, 4].

La información hidrológica a ser utilizada en el modelamiento y simulación de la operación del sistema hidráulico de EDEGEL S.A., corresponde a la necesidad de contar con series de caudales medios mensuales naturales en los puntos de interés de este sistema hidráulico como los embalses, centrales hidroeléctricas, tomas, etc., mostradas en la Figura 1, así como aquel que será utilizado por el proyecto de la C. H. de Huanza.

Para la obtención de esta información se efectuar un proceso de naturalización de caudales en la cuenca de los ríos Rímac y Santa Eulalia, en vista que el recurso hídrico, medido en las estaciones hidrométricas emplazadas en ella, tiene el efecto de las regulaciones de las 19 lagunas ubicadas en la cuenca alta del río Santa Eulalia y Marcapomacocha, respectivamente, así como también el efecto del embalse Yuracmayo ubicado sobre el río Blanco, afluente del río Rímac.

Se considera también los trasvases intercuencas efectuadas desde la cuenca de la laguna Marcapomacocha, perteneciente a la cuenca del río Mantaro, y el proyecto MARCA III, operando a partir del año 2000 [7].

El aprovechamiento de las aguas por el proyecto de la central hidroeléctrica de Huanza no alterará el régimen de la C.H. de Huinco, en las condiciones actuales, incluyendo el aprovechamiento de los recursos del proyecto MARCA III que comenzaron el año 2000, y las metas de funcionamiento del sistema hídrico del Rímac-Santa Eulalia y la laguna Marcapomacocha es de $25.0 \mathrm{~m}^{3} / \mathrm{s}$ en la estación de Chosica y de $8.0 \mathrm{~m}^{3} / \mathrm{s}$ en Sheque-Huinco. 
La ecuación considerada en la operación de los embalses del sistema hidráulico de la cuenca de los ríos Rímac y Santa Eulalia, es la ecuación de balance de masa, donde se toma en cuenta el caudal de ingreso, la evaporación, el caudal de salida y el almacenamiento producido en cada embalse, considerando a nivel mensual.

La ecuación general para la naturalización de las descargas de la laguna está dado por

$$
Q_{i}=Q_{s}+\frac{V_{i}-V_{i-1}}{\Delta t_{i}}+\frac{A_{i}\left(P_{i}-C E_{i}\right)}{\Delta t_{i}}+Q_{v}
$$

donde $Q_{i}$ es el caudal de ingreso en la laguna de embalse, $Q_{s}$ es el caudal de salida en las compuertas de fondo, $Q_{v}$ es el caudal vertido en el vertedero de demasías. Todos estos caudales están medido en $\mathrm{m}^{3} / \mathrm{s}$. Además $A_{i}$ es el área promedio del espejo de agua para el mes $i\left(\mathrm{~m}^{2}\right) ; P_{i}$ es la precipitación sobre el embalse para el mes $i(\mathrm{~m}) ; E_{i}$ es la evaporación desde el espejo de agua para el mes $i$ (m); $C$ es el factor de corrección de evaporímetro; $V_{i}$ es el volumen acumulado en el embalse para el mes $i\left(\mathrm{~m}^{3}\right)$; $V_{i-1}$ es el volumen acumulado en el embalse para el $i-1$ $\left(\mathrm{m}^{3}\right)$ y $\Delta t_{i}$ es el intervalo de tiempo para el mes $i(\mathrm{~s})$.

Pero en la hipótesis que la diferencia $\left(P_{i}-C E_{i}\right)$ sea pequeña o que está considerado implícitamente en la lectura del volumen almacenado, se tiene la siguiente simplificación

$$
Q_{i}=Q_{s}+\frac{V_{i}-V_{i-1}}{\Delta t_{i}}+Q_{v} .
$$

Pero en estiaje no hay salida por el vertedero, luego se tiene

$$
Q_{i}=Q_{s}+\frac{V_{i}-V_{i-1}}{\Delta t_{i}}
$$

Para todo el sistema hídrico a simular consideramos las siguientes áreas.

Ubicación del proyecto de la C.H. de Huanza. Según la demarcación política del país, el proyecto de la C.H. de Huanza se encuentra ubicado en la Provincia de Huarochiri, departamento de Lima, distrito de Huanza, dentro de cuyos linderos y de la propia comunidad de Huanza, se encuentran las obras civiles, razón por la que toma su nombre. Está localizado en la vertiente occidental de la Cordillera de los Andes, en la zona central del país, en la cuenca del río Santa Eulalia, afluente del río Rímac, captará las aguas de tres de los afluentes del río Santa Eulalia, -el Pallca, el Collque y el Saccsa-, en la cota 4030 m.s.n.m., ver Figura 3.

El punto de devolución de las aguas del Proyecto Huanza estará ubicado aguas arriba de las obras de captación de la central de Huinco representado por la captación y el embalse de regulación horaria de Sheque.

Las fuentes de agua del Proyecto son dos, la cuenca alta del Río Santa Eulalia y la Cuenca Transandina del río Mantaro trasvasada de la vertiente oriental. El área de drenaje de la cuenca de Santa Eulalia que será captada de los ríos Pallca y Collque, es de $199 \mathrm{~km}^{2}$. Parte de ella, $60.7 \mathrm{~km}^{2}$, es regulada en las lagunas de Huallunca, Canchis, Huasca, Quiusha y Piti, y la otra parte, $138.3 \mathrm{~km}^{2}$, no lo es.

En el área de drenaje que es trasvasada por el túnel transandino al Río Pallca, existen también sectores regulados en las lagunas de Marcapomacocha, Antacoto, Marcacocha y Sangrar que, en conjunto, alcanzan una superficie de $175 \mathrm{~km}^{2}$, y sectores no regulados, que son captados y transportados por el canal Tucto-Antacasha que abarcan una superficie de $70.7 \mathrm{~km}^{2}$. Adicionalmente, el Proyecto Marca III a partir del año 2000 adiciona los recursos que se captan de un área de drenaje de $102.6 \mathrm{~km}^{2}$, los que son embalsados y regulados en la Laguna de Antacoto.

Se contó con registros hidrométricos de los últimos 27 años en dos estaciones claves por su localización, así como información referente a los volúmenes mensuales de almacenamiento en los ya mencionados embalses de la cuenca de Santa Eulalia. Una de las estaciones es Milloc, que está situada a la salida del túnel transandino de trasvase y registra, por lo tanto, todos los caudales propios de esa cuenca, y la otra estación, Sheque, que está en el punto de captación de la C.H. de Huinco y mide los caudales trasvasados anteriormente mencionados más los de la cuenca procedente de Santa Eulalia.

Se ha considerado también a partir del año 2000 la incorporación del proyecto Marca III que incrementa en $1.48 \mathrm{~m}^{3} / \mathrm{s}$ los recursos de la derivación transandina. La incidencia de estos recursos en la C.H. de Huanza ha sido considerando solamente para el incremento de la generación de energía toda vez que el dimensionamiento ha sido deducido sin este proyecto.

Se determinaron también las necesidades de agua para riego en la situación actual, así como las demandas potenciales de las tierras susceptibles de irrigarse en base a lo cual se determinaron las reservas de agua necesarias en los sitios de captación para estos objetivos y se agregó el caudal mínimo que debiera dejarse para mantener el equilibrio ecológico. El total de estas reservas sumando los de Pallca y Collque arrojan un total de $300 \mathrm{lt} / \mathrm{s}$ los cuales se han descontado de las disponibilidades, obteniendo en definitiva los siguientes caudales aprovechables: 


\begin{tabular}{llll}
\hline Situación actual & $\begin{array}{l}Q_{\text {medio }}=6.38 \mathrm{~m}^{3} / \mathrm{s} \\
Q_{\text {firme }}=3.59 \mathrm{~m}^{3} / \mathrm{s}\end{array}$ & $-300 \mathrm{lt} / \mathrm{s}$ reserva & $\begin{array}{l}Q_{\text {medio }}=6.08 \mathrm{~m}^{3} / \mathrm{s} \\
Q_{\text {firme }}=3.29 \mathrm{~m}^{3} / \mathrm{s}\end{array}$ \\
\hline & & \\
$\begin{array}{l}\text { Situación actual }+ \\
\text { Marca III }\end{array}$ & $Q_{\text {medio }}=7.83 \mathrm{~m}^{3} / \mathrm{s}$ & $-300 \mathrm{lt} / \mathrm{s}$ reserva & $Q_{\text {medio }}=7.53 \mathrm{~m}^{3} / \mathrm{s}$ \\
\hline
\end{tabular}

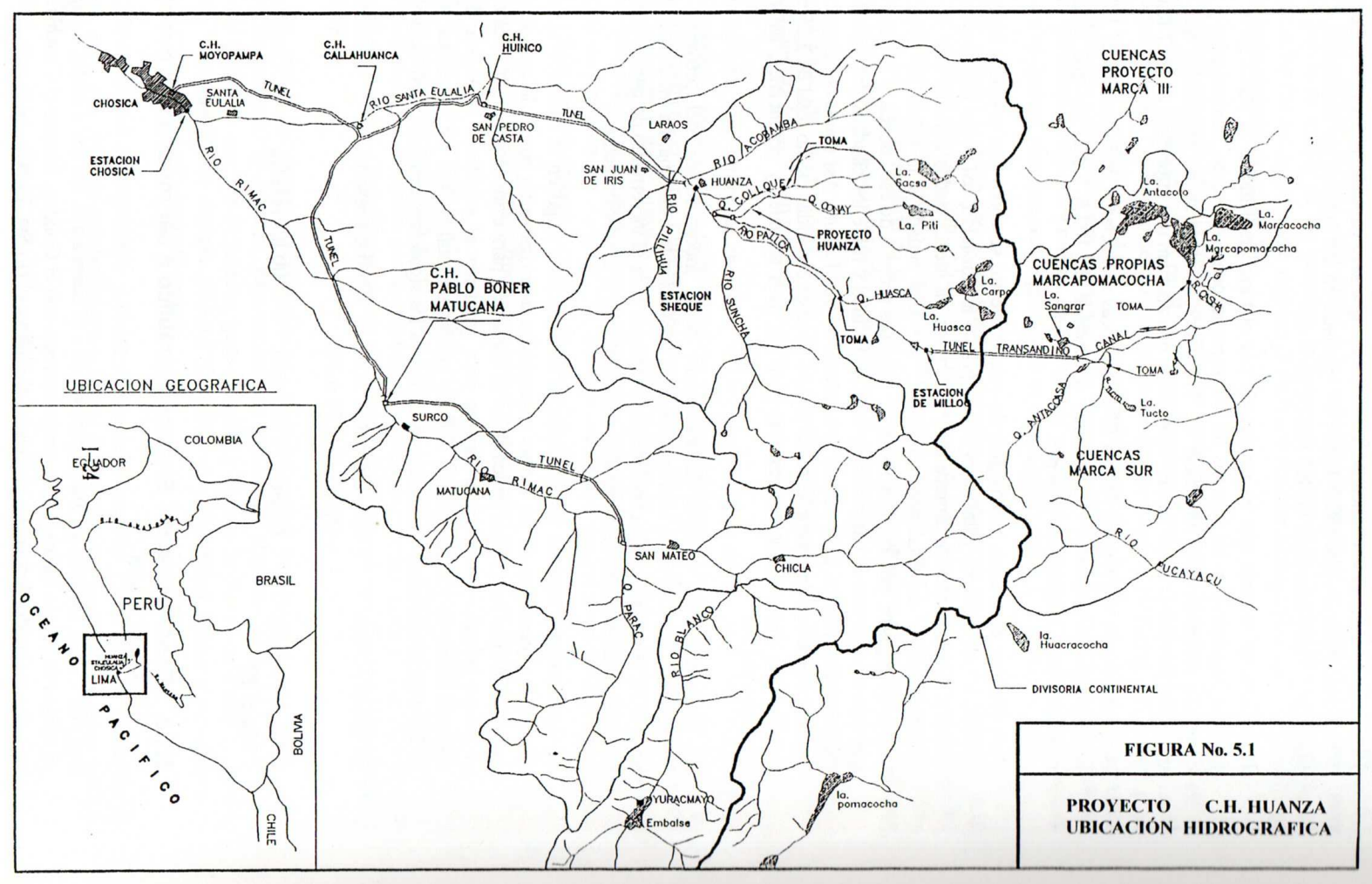

Figura 3: Ubicación hidrogeográfica del Proyecto de la Central Hidroeléctrica de Huanza.

Las principales obras hidráulicas de este sistema son: a) Represamiento de la cuenca del Río Sangrar mediante la presa de $21.5 \mathrm{~m}$ de altura máxima, $93 \mathrm{~m}$ de longitud en la coronación y 8 millones de $\mathrm{m}^{3}$ de capacidad de almacenamiento, recibiendo este vaso los aportes del río Jorococha mediante canal de $440 \mathrm{~m}$ de longitud. b) Canal colector Tucto-Antacasha-Cuevas-Sangrar, de $2.6 \mathrm{~km}$ de longitud de $6 \mathrm{~m}^{3} / \mathrm{s}$ de capacidad, revestido en piedra y concreto. c) Túnel Transandino de 10,122 m, con boca de entrada en las proximidades de la confluencia de los ríos Antacasha, Cuevas y Sangrar. d) Embalses de Antacoto, Marcapomacocha y Marcacocha con capacidad de almacenamiento de 61.0, 14.8 y 10.7 millones de $\mathrm{m}^{3}$, respectivamente. e) Túnel de descarga de la laguna de Antacoto, de $120 \mathrm{~m}$ dotado de compuertas para regulación de las descargas a la laguna de Marcapomacocha. f) Canal y toma de Antachupa, que conduce las descargas del río Antachupa y los caudales que rebosan de la laguna de Marcacocha a la laguna de Antacoto. g) Toma de Marcacocha, que regula los caudales de la laguna del mismo nombre y que desembocan a la laguna de Marcapomacocha. h) Canal de $8 \mathrm{~m}^{3} / \mathrm{s}$ de capacidad y $12.6 \mathrm{~km}$ de longitud que conduce los caudales regulados provenientes de la laguna de Marcapomacocha a la entrada del Túnel Transandino.

En la Tabla 1 se presenta la relación de lagunas y represamientos efectuados en la cuenca de los ríos Rimac y Santa Eulalia.

Las aguas de la derivación Marcapomacocha ingresan a la cuenca del Santa Eulalia a través del Túnel Transandino, que desemboca al Río Pallca en el paraje denominado Milloc a 4400 m.s.n.m., en el que existe una estación de aforos que mide sus descargas. Estas aguas discurren por el río Pallca y consecuentemente por la zona donde estaría la captación principal de la central proyectada de Huanza.

En el río San Mateo no existen lagunas importantes, por lo que en uno de sus tributarios, el río Blanco, se 
ha construido en 1998 el reservorio de Yuracmayo, de 50 MMC de capacidad total y 43 MMC de volumen útil.

Se obtienen los siguientes registros de información: a) Cuenca alta Río Santa Eulalia. Como ya se ha dicho, los ríos Pallca, Collque y Sacsa se encuentran en la cuenca alta del Santa Eulalia que fluye hasta el punto de origen del río, esto es, la estación y embalse de Sheque. Estos registros datan de 1965; año en el que se inició la operación de la central de Huinco. Los datos de la estación Sheque incluyen los ingresos de la derivación de Marcapomacocha, cuya medición se realiza en la estación de Milloc, ubicada a la salida del túnel y también mantenida y controlada por las Empresas Eléctricas primero, y por Electrolima después.

No existen datos sobre la operación de cada laguna de regulación de la cuenca propia, ya que la información sobre los volúmenes mensuales embalsados a fines de cada mes, sólo se inicia a partir de 1967.

Respecto a la información pluviométrica, los únicos datos continuos y confiables son los de la estación de Milloc y los de la estación Carampoma, ubicada en la parte inferior de la cuenca. Las demás estaciones pluviométricas han interrumpido su funcionamiento, o no cuentan con información confiable. b) Cuenca Media río Santa Eulalia. Los caudales en la cuenca del río Santa Eulalia han sido controlados, entre 1950 y 1964, en la estación hidrométrica Austisha y, a partir de 1965 a la fecha, en la estación hidrométrica Sheque. c) Cuenca Río Rímac. En la estación de Chosica, que registra el escurrimiento de toda la cuenca del Rímac incluyendo la del Sta. Eulalia, se dispone de información desde 1922 hasta la fecha. Los datos de 1922 a 1967 se registraron en diferentes secciones de la zona Chacrasana-Puente Ñaña y en la toma de Yanocoto, en base a limnimetros y aforos diarios; y, a partir de 1967, el SENAMHI instaló una estación limnigráfica en Chosica, la misma que controla y cuyos datos procesa. Toda la información ha sido debidamente analizada y procesada por la ex ONERN, fuente que ha sido utilizada para este estudio. d) Cuenca del río San Mateo. Los caudales en la cuenca del río San Mateo han sido controlados, entre 1957 y 1974, en la estación hidrométrica Anyahuari (Surco) y, desde 1972 a la fecha en la estación hidrométrica Tamboraque. Otras estaciones hidrométricas de gran importancia en esta cuenca son las del río Blanco en la cercanía de la presa Yuracmayo, que cuenta con registros.

Se dispone de datos generados de caudales de ingreso a los reservorios procedentes de su propia cuenca, producidos en los estudios que SEDAPAL lleva a cabo para efectos del Proyecto Marca III. Y así mismo se dispone de datos de caudales derivables por el colector Antacasa - Tucto, generados para efectos del proyecto Marca Sur, por la firma C. y A. Consultores y Asesores Asociados.

Igualmente, se cuenta con datos generados de los caudales que aportará el proyecto Marca III al Sistema Marcapomacocha, obtenidos para este proyecto por la Empresa
Grana y Montero Ingeniería S.A. (GMI).

Las metas a cumplir por el Sistema Hidráulico RímacSanta Eulalia, SEDAPAL, en su Plan Referencial, considera que se debe tener una dotación en Chosica de 25.0 $\mathrm{m}^{3} / \mathrm{s}$ al $95 \%$ que suministre agua para abastecimiento poblacional y para la poca agricultura existente y para el caudal ecológico del río en la parte que cruza la ciudad.

En lo que respecta a la central de Huinco, en los 27 años de funcionamiento registran un caudal medio de $11.33 \mathrm{~m}^{3} / \mathrm{s}$; de $6.5 \mathrm{~m}^{3} / \mathrm{s}$ al $95 \%$ y de $7.20 \mathrm{~m}^{3} / \mathrm{s}$ al $90 \%$. Con el aporte de Marca III de $1.48 \mathrm{~m}^{3} / \mathrm{s}$, con la mayor capacidad de regulación, sin incrementar mayormente la evaporación y con una adecuada regla de operación es posible alcanzar los $8.00 \mathrm{~m}^{3} / \mathrm{s}$ como caudal firme al $95 \%$ de duración, manteniendo la suma de los medios 12.8 como caudal medio multianual del nuevo sistema en Sheque.

En tal consideración, las metas de funcionamiento del sistema hídrico del Rímac-Marcapomacocha, con la incorporación de Marca III y considerando la regulación del río Blanco en Yuracmayo, es de $25.0 \mathrm{~m}^{3} / \mathrm{s}$ en la estación de Chosica y de $8.0 \mathrm{~m}^{3} / \mathrm{s}$ en Sheque-Huinco. El esquema hídrico simplificado para esta situación se muestra en la Figura 4.

Los caudales afluentes a este sistema son: a) Sistema de derivación Marcapomacocha-Túnel Trasvase. Ingreso al reservorio de Mancacoto que forman parte el Proyecto Marca III, $Q_{2}=1.48 \mathrm{~m}^{3} / \mathrm{s}$. La cuenca propia de Marcapomacocha, $Q_{1}=2.29 \mathrm{~m}^{3} / \mathrm{s}$, Laguna Sangrar, $0.60 \mathrm{~m}^{3} / \mathrm{s}$, colector Antacasa-Tucto $Q_{3}=0.75 \mathrm{~m}^{3} / \mathrm{s}$, filtración en el túnel, $Q_{4}=1.00 \mathrm{~m}^{3} / \mathrm{s}$. b) Los caudales de la Cuenca de Santa Eulalia que se consideran los ingresos de las lagunas de la cuenca de Huanza, $Q_{5}=0.72 \mathrm{~m}^{3} / \mathrm{s}$, de las lagunas fuera de la cuenca de Huanza, $Q_{7}=0.69$ $\mathrm{m}^{3} / \mathrm{s}$; los escurrimientos no regulados de la cuenca de Huanza, $Q_{6}=1.27 \mathrm{~m}^{3} / \mathrm{s}$, y fuera de la cuenca de Huanza, $Q_{8}=2.13 \mathrm{~m}^{3} / \mathrm{s}$. c) Ingreso al reservorio Yuracmayo, $Q_{9}=2.13 \mathrm{~m}^{3} / \mathrm{s}$. d) Caudales de la cuenca Rímac sin Sheque (Huinco) y sin Yuracmayo, $Q_{10}=17.07 \mathrm{~m}^{3} / \mathrm{s}$.

Como se ha dicho en párrafos anteriores, no hemos tenido acceso a mediciones a la entrada del túnel transandino, por lo que no es posible deducir directamente los caudales correspondientes a las filtraciones en dicho túnel; se asumió un caudal medio correspondiente a las filtraciones del orden de $1 \mathrm{~m}^{3} / \mathrm{s}$, valor que es adoptado, asumiendo una distribución constante a lo largo del año.

En cuanto a los caudales provenientes de Yuracmayo, son los registrados en la estación de río Blanco y los del Rímac, los medidos en la estación Chosica, descontando los de Sheque y Yuracmayo.

\section{Modelo de simulación de la operación del sistema hidráulico: Cuenca Río Rímac-Sta. Eulalia}

Para analizar el funcionamiento del sistema hidráulico representado en el esquema simplificado de la Figura 4, y 
alcanzar las metas señaladas en el ítem 3.3, se formuló un modelo de simulación, el mismo que opera con las siguientes características: a) El periodo de análisis es 27 años en base a la serie histórica de 1978 al 2005, con datos a nivel mensual. b) Los ingresos de agua al sistema son los naturales (no regulados) que se muestra en el esquema simplificado Figura 4. c) La demanda del modelo global será de $25.40 \mathrm{~m} 3 / \mathrm{s}$ que necesita cubrir el sistema para satisfacer la necesidad de agua potable, agricultura en Chosica y ecológico. d) En primera instancia, se verá la medida en que los caudales del Rímac sin regulación debajo de Sheque y Yuracmayo cubren esta demanda, determinando así los saldos a cubrir. Estos saldos serán el primer punto de control del sistema (Q7). e) Para un primer análisis, se ha tomado una tasa constante de 0.70 de la evaporación máxima para los embalses de Yuracmayo y Antacoto, y no se ha considerado la de los embalses del Sta. Eulalia, pues ello ya se hizo al reconstruir el régimen natural de caudales. f) Otro punto de control del sistema será el mantenimiento de un mínimo de $8 \mathrm{~m} 3 / \mathrm{s}$ en Sheque para energía, para la generación en Huinco (Q6). g) El primer paso en la simulación es la operación del reservorio de $\mathrm{Yu}$ racmayo, para cubrir los saldos de Chosica estableciéndose en esta operación nuevos déficits (Q8). h) Los déficits resultantes de la operación del reservorio de Yuracmayo con demandas en Chosica, se compara con las demandas mínimas en Sheque, adoptándose las de mayor valor, como las demandas a cubrir (Q12) con las nuevas disponibilidades en Sheque. i) El cubrimiento de las demandas de Sheque se hace en primer lugar con los caudales no regulados que pasan por ese punto (Q10); estableciéndose así nuevos saldos a cubrir desde los reservorios (Q13). Los caudales naturales (Q11) que ingresan a los reservorios serán regulados en estos pare egresarlos según los saldos establecidos (Q13).

De este proceso se determinan los déficits no cubiertos (Q14) y los excedentes que pasan por Huinco (Q15). Los resultados de la simulación de Marca III suministrará en Chosica un caudal de $25 \mathrm{~m} 3 / \mathrm{s}$ al $97 \mathrm{j}$ ) Los caudales que pasan por Sheque con Marca III y después de la operación de los reservorios se determinaran sumando a las demandas totales establecidas en Sheque (Q12) más excedentes (Q15) y descontando los déficits (Q14). k) Los Caudales que pasan por Huanza se deducen de los de Sheque en forma similar a la situación actual. I) Los déficits finales en Huinco y Chosica se establecerán comparando los caudales que pasan por Huinco (Q16) con las metas en Sheque (Q6) de $8.0 \mathrm{~m} 3 / \mathrm{s}$ y con los déficits en el Rimac después de Yuracmayo (Q8), estos déficits se expresarán en \% de meses no cubiertos en relación del total de meses de la serie.

\section{Resultados}

Se ha elaborado un modelo de simulación del sistema hidráulico-hidrológico en modo "spreadsheet", el cual considera como puntos de interés las bocatomas de captación de las centrales hidroeléctricas de EDEGEL S.A., las características técnicas de las centrales hidráulicas, y como caudales de ingreso a estos puntos las series de caudales medios mensuales naturales de ingreso a los 19 embalses de regulación de la cuenca alta del río Santa Eulalia y laguna Marcapomacocha-cuenca del río Mantaro, los caudales de vaporación del espejo de agua de los embalse y los caudales de infiltración, y los caudales medios mensuales naturales aportados por las cuencas intermedias entre los embalses y las bocatomas o puntos de interés, las demandas de riego y la gran demanda de agua potable para las ciudades de Lima y Callao.

Los resultados indican que se cumplen las metas para Chosica, en un nivel de excedencias del $95 \%$ sobre 25 $\mathrm{m}^{3} / \mathrm{s}$, y para Huinco, los $8 \mathrm{~m}^{3} / \mathrm{s}$ al $96 \%$ de duración. El caudal promedio es $12.51 \mathrm{~m} 3 / \mathrm{s}$, que significa un incremento de energía de aproximadamente $14.5 \%$, con caudales medios mensuales para el período simulado 1978/79 a 2004/05. En el cuadro siguiente se presenta un resumen estadístico de la serie de caudales mencionado anteriormente.

\begin{tabular}{cccccccccccccc}
\hline Año & Oct & Nov & Dic & Ene & Feb & Mar & Abr & May & Jun & Jul & Ago & Set & Prom \\
\hline Prom & 11.58 & 9.92 & 9.42 & 11.98 & 16.08 & 20.06 & 14.52 & 9.49 & 8.91 & 11.76 & 13.71 & 12.69 & 12.51 \\
Máx & 15.71 & 14.11 & 16.13 & 23.54 & 31.08 & 40.68 & 25.43 & 14.50 & 16.15 & 16.71 & 19.09 & 17.94 & 16.12 \\
Mín & 6.13 & 4.18 & 4.44 & 7.30 & 7.22 & 8.00 & 7.74 & 8.00 & 7.74 & 8.00 & 8.00 & 7.74 & 10.24 \\
\hline
\end{tabular}

En Huanza que viene a ser dentro del sistema hidráulico, un punto de control dependiente de la operación en conjunto de todo el sistema, para alcanzar las metas de Huinco y Chosica, se obtiene como resultado un caudal medio de $7.83 \mathrm{~m}^{3} / \mathrm{s}$ con un firme al $95 \%$ de $3.71 \mathrm{~m}^{3} / \mathrm{s}$, de la serie de 27 años con caudales medios mensuales para el período 1978/769 a 2004/05. En el cuadro siguiente muestra un resumen estadístico de la serie de caudales mencionado anteriormente. 


\begin{tabular}{cccccccccccccc}
\hline Año & Oct & Nov & Dic & Ene & Feb & Mar & Abr & May & Jun & Jul & Ago & Set & Prom \\
\hline Prom & 8.65 & 7.16 & 5.42 & 5.60 & 7.44 & 9.29 & 7.82 & 6.07 & 6.84 & 9.17 & 10.72 & 9.82 & 7.83 \\
Máx & 13.18 & 12.92 & 13.90 & 12.72 & 17.42 & 24.19 & 16.01 & 11.74 & 14.00 & 14.84 & 17.16 & 15.93 & 10.87 \\
Mín & 4.25 & 2.87 & 2.70 & 3.32 & 3.88 & 3.94 & 3.89 & 4.08 & 3.60 & 3.51 & 3.29 & 3.54 & 5.78 \\
\hline
\end{tabular}

\section{Discusión}

Uno de los resultados obtenidos de la simulación multipropósito de la operación del sistema hidráulico RímacSanta Eulalia en condiciones actuales incluyendo el proyecto Marca III, es el de haber obtenido una serie de caudales mensuales con un caudal medio multianual de $12.51 \mathrm{~m}^{3} / \mathrm{s}$ a ser usado por la C. H. de Huinco, con una producción de energía anual de 1157.14 Gwh.

Históricamente, el caudal medio multianual utilizado por la central de Huinco es de $11.16 \mathrm{~m}^{3} / \mathrm{s}$ sin considerar el aporte de MARCA III, con una producción de energía anual del orden de $1010.46 \mathrm{Gwh}$, por consiguiente se ha obtenido un $14.5 \%$ más de generación de energía en la central de Huinco.

Los estudios del proyecto de la C. H. de Huanza han determinado que el caudal medio multianual a ser utilizado es de $7.83 \mathrm{~m}^{3} / \mathrm{s}$, su potencia instalada de $79.4 \mathrm{Mw}$, su capacidad de generación es de $336 \mathrm{Gwh}$. Como resultado de la simulación de la simulación realizada se ha obtenido una serie de 27 años con un caudal medio multianual de $7.83 \mathrm{~m}^{3} / \mathrm{s}$ para Huanza, pero si se descuenta $300 \mathrm{l} / \mathrm{s}$ por reserva de agua para la agricultura y el caudal ecológico a ser dejados en la cuenca de Pallca y Collque se tendrá un caudal final de: $7.83-0.300=7.53 \mathrm{~m}^{3} / \mathrm{s}$, obteniéndose una generación de energía anual de $331.80 \mathrm{Gwh}$. Luego la diferencia encontrada es del orden del $1.5 \%$, considerándose esta diferencia como mínima y aceptando como $v$ 'alido los resultados obtenidos en la simulación efectuada.

En lo que respecta al cubrimiento del caudal para la agricultura, la fuente de agua potable y el aporte ecológico en el punto de control de Chosica, es decir, contar con un caudal total de $25 \mathrm{~m}^{3} / \mathrm{s}$, este es cubierto en un $95 \%$ del tiempo durante el período simulado de 27 años.

El modelo planteado para efectuar la simulación de la operación del sistema hidráulico de la cuenca del río Rímac-Santa Eulalia da resultados aceptables, tal como lo confirma lo expuesto en los párrafos anteriores, y por lo tanto puede ser usado para evaluar futuros proyectos de centrales hidroeléctricas y el emplazamiento de nuevos embalses en esta cuenca, con solo efectuar pequeñas modificaciones en su estructura.

\section{Conclusiones}

Las condiciones actuales de simulación de la operación en el modelo planteado para la cuenca del río Rímac-Sta. Eulalia, tiene como únicos puntos de control, el caudal a ser usado por el proyecto de la C.H. Huanza, la cuantificación del el recurso hídrico a la altura del embalse horario de Sheque, lugar donde se encuentra la toma de la C.H. Huinco, y finalmente el caudal en el sitio de emplazamiento de la estación hidrométrica Chosica. Los resultados obtenidos de la simulación de la operación del sistema hidráulico del Rímac-Santa Eulalia son aceptables, en vista que el modelo a dado respuesta a las premisas bajo las cuales ha operado, como es el caso de garantizar un caudal medio multianual de $12.51 \mathrm{~m}^{3} / \mathrm{s}$ a ser utilizado por la C. H. de Huinco y el de cubrir con un $95 \%$ del tiempo simulado (27 años) el caudal de $25 \mathrm{~m}^{3} / \mathrm{s}$ en la estación de Chosica, recurso utilizado para cubrir la demanda agrícola, poblacional y ecológico de Lima. El modelo de simulación planteado para la cuenca del río Rímac-Santa Eulalia, puede ser usado para la evaluación de cualquier proyecto hidroeléctrico, embalse de regulación y política de abastecimiento de agua para uso agrícola y poblacional, con solo efectuar pequeñas modificaciones en su estructura. Actualmente, existe incertidumbre en el abastecimiento del Gas Natural de Camisea como solución a corto plazo y mediano plazo para el cubrimiento de la demanda de energía del Sistema Eléctrico Interconectado Nacional (SEIN), al cual pertenece la cuenca del río Rímac-Santa Eulalia. Por lo tanto cualquier nuevo proyecto de generación hidráulica propuesto dentro de esta cuenca puede ser altamente rentable, en vista que se encuentra cerca del centro de mayor demanda de energía del SEIN, que es Lima y Callao, y que tendría a favor el hecho de que toda la infraestructura civil de afianzamiento hídrico, como es el caso de represamiento de lagunas, transvases intercuencas, etc., ya existen. Por todo lo expuesto anteriormente, el costo del proyecto hidroeléctrico sería mucho menor, y todo ello puede ser evaluado por medio del modelo planteado para efectuar la simulación de la operación del sistema hidráulico de la cuenca del río Rímac-Santa Eulalia. 


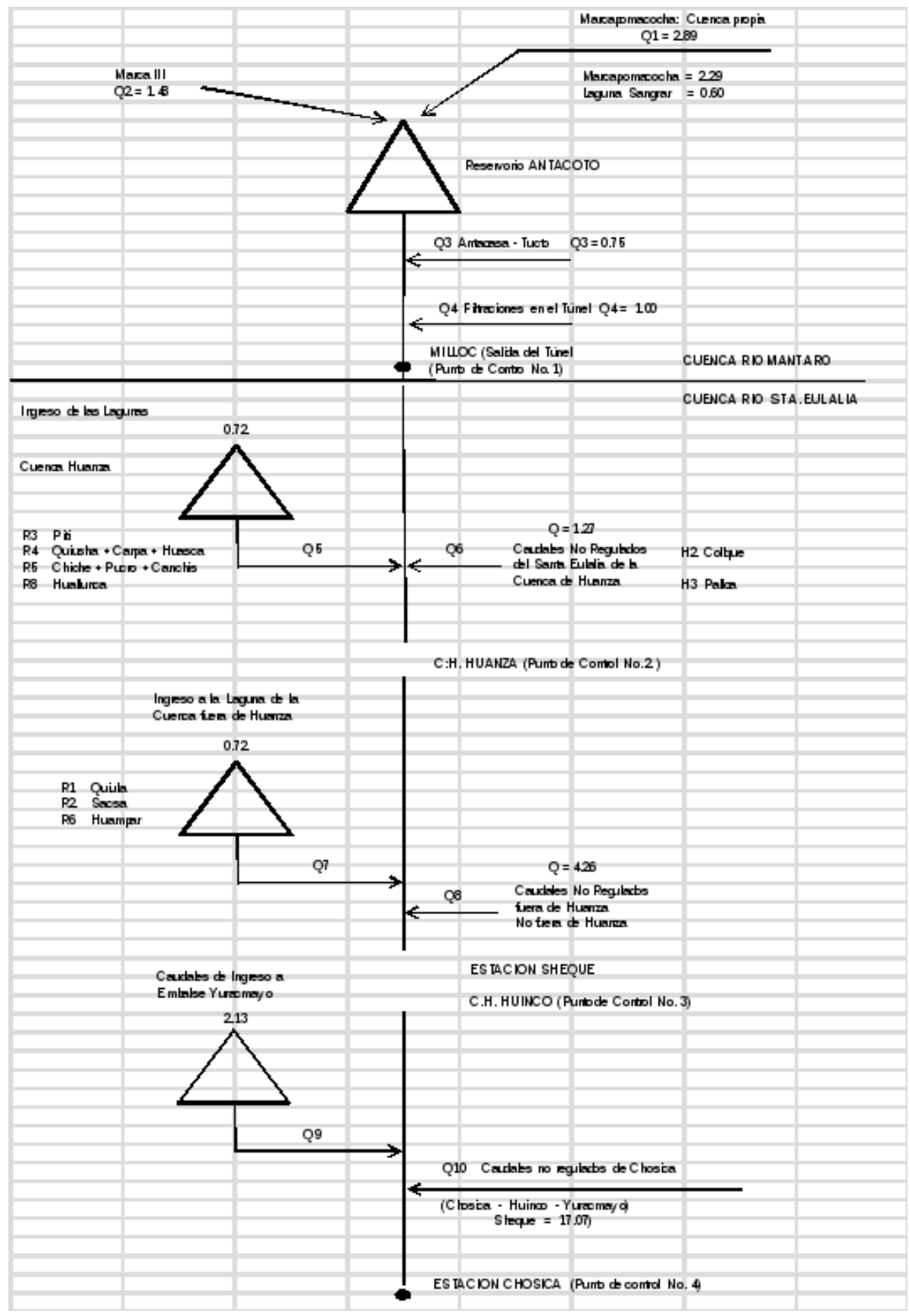

Figura 4: Esquema simplificado del sistema hidráulico actual de la cuenca Rímac-Santa Eulalia mostrando la disponibilidad hídrica con el Proyecto Marca III 


\begin{tabular}{|c|c|c|c|c|c|c|c|c|c|c|}
\hline \multirow[b]{2}{*}{$\mathrm{N}^{\mathrm{o}}$} & \multirow[b]{2}{*}{ Laguna } & \multirow[b]{2}{*}{$\begin{array}{l}\text { Área de } \\
\text { la cuenca } \\
\left(\mathrm{km}^{2}\right)\end{array}$} & \multirow[b]{2}{*}{$\begin{array}{l}\text { Volumen } \\
\text { máximo } \\
\text { embalse } \\
(\mathrm{MMC})\end{array}$} & \multirow[b]{2}{*}{$\begin{array}{l}\text { Volumen } \\
\text { útil } \\
(\mathrm{MMC})\end{array}$} & \multirow[b]{2}{*}{$\begin{array}{l}\text { Espejo } \\
\text { de agua } \\
\left(\mathrm{km}^{2}\right)\end{array}$} & \multicolumn{4}{|c|}{ Características de la presa } & \multirow[b]{2}{*}{$\begin{array}{l}\text { Año de } \\
\text { ejecución }\end{array}$} \\
\hline & & & & & & Tipo & $\begin{array}{l}\text { Altura } \\
(\mathrm{m})\end{array}$ & $\begin{array}{l}\text { Longitud } \\
\text { de coro- } \\
\text { nación } \\
(\mathrm{m})\end{array}$ & $\begin{array}{l}\text { Ancho } \\
\text { de coro- } \\
\text { nación } \\
(\mathrm{m})\end{array}$ & \\
\hline 1 & Quiula & 7.0 & 1.80 & & & mampo & 5.6 & 173 & 1.0 & 1940 \\
\hline 2 & Sacsa & 33.7 & 16.75 & 14.89 & 1.63 & mampo & 13.0 & 140 & 1.2 & 1875 \\
\hline 3 & Quisha & 8.3 & 9.15 & 8.67 & 0.65 & $\begin{array}{l}\text { arco- } \\
\text { gravedad }\end{array}$ & 16.6 & 51 & 1.5 & 1875 \\
\hline 4 & Piti-Culi & 7.1 & 7.1 & 6.5 & 1.02 & mampo & 9.9 & 172 & 1.0 & 1875 \\
\hline 5 & Carpa & 18.5 & 21.2 & 17.8 & 1.35 & $\begin{array}{l}\text { arco- } \\
\text { gravedad }\end{array}$ & 16.4 & 50 & 1.5 & 1875 \\
\hline 6 & Huasca & 27.0 & 6.55 & 6 & 0.9 & mampo & 9.0 & 63 & 1.0 & 1875 \\
\hline 7 & Misha & 3.3 & 0.67 & 0.65 & 0.13 & mampo & 5.3 & 12 & 1.5 & 1875 \\
\hline 8 & Chiche & 7.0 & 2.60 & 2.22 & 0.35 & mampo & 9.5 & 61 & 2.0 & 1930 \\
\hline 9 & Pucro & 8.0 & 2.08 & 1.96 & 0.22 & mampo & 10.3 & 22 & 1.5 & 1875 \\
\hline 10 & Canchis & 26.0 & 2.24 & 2.08 & 0.4 & mampo & 10.3 & 54 & 1.0 & 1825 \\
\hline 11 & Huallunca & 6.4 & 1.00 & 1.6 & 0.2 & tierra & 12.1 & 124 & 3.5 & 1940 \\
\hline 12 & Huampar & 8.2 & 3.8 & 3.33 & 0.52 & mampo & 9.8 & 101 & 2.5 & 1926 \\
\hline 13 & Huachua & 16.1 & 5.6 & 4.98 & 0.6 & mampo & 13.9 & 228 & 1.5 & 1875 \\
\hline 14 & Pirhua & 2.3 & 1.0 & 0.95 & 0.15 & mampo & 9 & 70 & 1.0 & 1876 \\
\hline 15 & manca & 7.1 & 1.73 & 1.57 & 0.13 & mampo & 14.8 & 105 & 1.5 & 1875 \\
\hline
\end{tabular}

Tabla 1: Características de las Obras de Regulación de la cuenca de los ríos Rímac y Santa Eulalia. Elaboración propia con datos de EDEGEL S.A. 


\section{Referencias}

[1] MIMEM, Plan Referencial de Electricidad 2008-2017, Dirección General de Electricidad del Ministerio de Energía y Minas (2008).

[2] Ray K. Linsley y Joseph B. Franzini; Ingeniería de los Recursos Hidráulicos, C.E.C.S.A., México (1984).

[3] Otto J. Helweg, Recursos Hidráulicos Planeación y Administración, Limusa y Grupo Noriega Editores, México (1992).

[4] CyA-PROHISA, Estudio de Factibilidad Proyecto Central Hidroeléctrica Huanza, Harza Engineering Company Internacional, Lima (2006).
[5] OSINERMIN, Estudio técnico económico de determinación de precios de potencia y energía para la fijación tarifaria de mayo 2012 a abril 2013, OSINERMINOTERG, Lima (2012).

[6] Comisión de Tarifas Eléctricas, CTE; Modelo de costos marginales de electricidad del Sistema Interconectado Centro-Norte, SICN, SA\&E S.A.-ENGENHO, Lima (1997).

[7] SEDAPAL, Estudio definitivo del Proyecto de Afianzamiento Marcapomacocha (MARCA III), Graña y Montero Ingeniería S.A., GMI, Lima (1996). 